(C) The Authors 2019. This is an Open Access article, distributed under the terms of the Creative Commons Attribution licence (http:// creativecommons.org/licenses/by/4.0/), which permits unrestricted re-use, distribution, and reproduction in any medium, provided the original work is properly cited.

\title{
Effect of lifelong carnitine supplementation on plasma and tissue carnitine status, hepatic lipid metabolism and stress signalling pathways and skeletal muscle transcriptome in mice at advanced age
}

\author{
Uzman B. Cheema, Erika Most, Klaus Eder and Robert Ringseis* \\ Institute of Animal Nutrition and Nutrition Physiology, Justus-Liebig-University Giessen, Heinrich-Buff-Ring 26-32, \\ 35392 Giessen, Germany \\ (Submitted 15 November 2018 - Final revision received 27 February 2019 - Accepted 13 March 2019 - First published online 10 June 2019)
}

\begin{abstract}
While strong evidence from clinical studies suggests beneficial effects of carnitine supplementation on metabolic health, serious safety concerns associated with carnitine supplementation have been raised from studies in mice. Considering that the carnitine doses in these mice studies were up to 100 times higher than those used in clinical studies, the present study aimed to address possible safety concerns associated with long-term supplementation of a carnitine dose used in clinical trials. Two groups of NMRI mice were fed either a control or a carnitine-supplemented diet ( $1 \mathrm{~g} / \mathrm{kg}$ diet) from weaning to 19 months of age, and parameters of hepatic lipid metabolism and stress signalling and skeletal muscle gene expression were analysed in the mice at 19 months of age. Concentrations of free carnitine and acetylcarnitine in plasma and tissues were higher in the carnitine than in the control group $(P<0.05)$. Plasma concentrations of free carnitine and acetylcarnitine were higher in mice at adult age (10 and 15 months) than at advanced age (19 months) $(P<0 \cdot 05)$. Hepatic mRNA and protein levels of genes involved in lipid metabolism and stress signalling and hepatic and plasma lipid concentrations did not differ between the carnitine and the control group. Skeletal muscle transcriptome analysis in 19-month-old mice revealed only a moderate regulation between carnitine and control group. Lifelong carnitine supplementation prevents an age-dependent impairment of plasma carnitine status, but safety concerns associated with long-term supplementation of carnitine at doses used in clinical trials can be considered as unfounded.
\end{abstract}

\section{Key words: Carnitine supplementation: Aged mice: Liver: Skeletal muscle}

L-Carnitine has long been known to be vital to all cells containing mitochondria due to its ability to shuttle long-chain activated fatty acids (acyl-CoA) as acylcarnitines from the cytosol into the mitochondrial matrix, where fatty acid $\beta$-oxidation takes place $^{(1)}$. However, carnitine is also involved in carbohydrate oxidation because it is also required for the efflux of acyl groups as acylcarnitines out of mitochondria, thereby reducing the intramitochondrial acetyl-CoA:CoA ratio ${ }^{(2)}$. As a consequence, acetyl-CoA-mediated inhibition of the activity of pyruvate dehydrogenase, a key metabolic enzyme of glucose utilisation, is relieved and glucose oxidation is stimulated ${ }^{(3,4)}$. This explains, at least partially, that a reduced carnitine status in diabetic subjects ${ }^{(5-7)}$ is associated not only with impaired fatty acid oxidation but also with perturbations of glucose utilisation and insulin sensitivity. In line with this, a large number of studies in humans and animals revealed that supplementation of carnitine or carnitine derivatives (e.g. acetylcarnitine and propionylcarnitine) improves the carnitine status and causes an improvement of whole-body glucose tolerance and insulin sensitivity of insulin-dependent tissues such as skeletal muscle and heart, in particular during insulin-resistant states ${ }^{(8)}$. In addition, clinical studies even demonstrated that myocardial carnitine deficiency is associated with cardiomyopathy ${ }^{(9,10)}$, and carnitine supplementation restores tissue levels of carnitine and improves heart function $^{(9,11)}$. Moreover, a significant number of clinical studies showed an improvement of quality of life measures, nutritional status and body condition and a reduction of fatigue-related symptoms in patients with chronic diseases such as cancer, chronic kidney disease, HIV, or hepatic encephalopathy due to carnitine supplementation ${ }^{(12-16)}$. Such patients frequently exhibit reduced plasma carnitine levels ${ }^{(17-19)}$ as a consequence of different reasons, such as a reduced food intake, impaired

Abbreviations: Aldh9a1, aldehyde dehydrogenase 9 family member A1; Ankrd2, ankyrin repeat domain $2 ; B 4 g a l n t 2, \beta-1,4-N$-acetylgalactosaminyltransferase 2 ; $\gamma$-BB, $\gamma$-butyrobetaine; Bbox1, $\gamma$-butyrobetaine hydroxylase 1; BW, body weight; ER, endoplasmic reticulum; FC, fold change; GO, gene ontology; GSEA, gene set enrichment analysis; Octn2, organic cation transporter 2; Olfr, olfactory receptor; qPCR, quantitative real-time PCR; SREBP, sterol regulatory element-binding protein.

* Corresponding author: R. Ringseis, email robert.ringseis@ernaehrung.uni-giessen.de 
intestinal carnitine absorption as a side effect of pharmacotherapy and chemotherapy and loss of carnitine during hemodialysis as in the case of chronic kidney disease patients.

Despite this large body of evidence from clinical studies ${ }^{(8-16)}$ that an improvement of carnitine status has beneficial effects on metabolic health, serious safety concerns with carnitine supplementation have been raised based on the recent observation that long-term carnitine supplementation (from weaning to 5 months of age) in a transgenic mouse model of atherosclerosis (Apo $\mathrm{e}^{-/-}$mice) promotes atherosclerosis through gut microbiotadependent formation of trimethylamine and subsequent hepatic conversion into proatherogenic trimethylamine- $N$-oxide ${ }^{(20)}$. In addition, it has been found that carnitine supplementation to normal mice for 3 months induces oxidative stress, liver inflammation, liver lipid accumulation and even liver damage as well as hypercholesterinaemia $^{(21)}$. Regarding these reports, the widespread use of carnitine supplements, which are easily distributed over the counter, in both the healthy and ill population might be seen critically. However, it has to be mentioned that the carnitine dose administered to the mice in the aforementioned studies was extremely high. In that study ${ }^{(21)}$, about $200 \mathrm{mg}$ carnitine was taken up daily per mouse from carnitine-supplemented drinking water which related to approximately $4000 \mathrm{mg} / \mathrm{kg}$ body weight (BW). The carnitine dose in the study with the $A p o e^{-/-}$mice ${ }^{(20)}$ was even greater than $4000 \mathrm{mg} / \mathrm{kg} \mathrm{BW}$ considering a carnitine concentration of $1.3 \%$ in the drinking water and an estimated daily water intake of $10 \mathrm{ml}$ by a 5-month-old mouse consuming 3-4 $\mathrm{g}$ feed per $\mathrm{d}$. By contrast, in clinical trials, the daily amount of carnitine provided to human subjects is typical between 2 and $6 \mathrm{~g}^{(22,23)}$, which relates to a carnitine dose of $30-90 \mathrm{mg} / \mathrm{kg} \mathrm{BW}$ for a person weighing $65 \mathrm{~kg}$. It might be argued therefore that the detrimental outcomes of carnitine supplementation in the aforementioned mice studies are due to the extremely high dose of carnitine administered.

Thus, in order to address whether safety concerns are founded under conditions of long-term supplementation of a carnitine dose used in clinical trials, the present study aimed to investigate the effect of almost lifelong daily administration of a carnitine-supplemented diet $(1 \mathrm{~g} / \mathrm{kg}$ diet) to mice from weaning to 19 months of age. Considering a BW of $50 \mathrm{~g}$ for an adult mice and a daily feed intake of $5 \mathrm{~g}$, the daily intake of this diet results in a daily carnitine dose of $100 \mathrm{mg} / \mathrm{kg} \mathrm{BW}$ which better reflects the dose used in clinical trials. In order to evaluate possible detrimental effects of carnitine supplementation, we investigated parameters of lipid metabolism and stress signalling pathways (endoplasmic reticulum (ER) stress-induced unfolded protein response, NF- $\mathrm{BB}$, nuclear factor-E2 related factor 2) in the liver and performed a genome-wide transcriptome analysis of skeletal muscle in the mice at advanced age.

\section{Methods}

\section{Animals and diets}

The animal experiment was approved by the local Animal Care and Use Committee (Regierungspräsidium Giessen; permission no. V 54 - 19 c 2015 h 02 GI 19/3 kTV 12/2018). All experimental procedures described followed established guidelines for the
Table 1. Composition of the basal natural ingredient diet

\begin{tabular}{lc}
\hline Component & $\mathrm{g} / \mathrm{kg}$ diet \\
\hline Wheat & 429.5 \\
Maize & 252 \\
Soyabean extraction meal (44 \% crude protein) & 200 \\
Wheat bran & 40 \\
Soyabean oil & 28 \\
Oats & 20 \\
Calcium hydrogen phosphate dihydrate & 11 \\
Mineral-vitamin mix & 6 \\
Calcium carbonate & 6 \\
DL-Methionine & 4.5 \\
L-Lysine-HCl & 1.5 \\
Choline chloride & 1.5 \\
\hline
\end{tabular}

* The mineral-vitamin mix provided the following per $\mathrm{kg}$ of diet: $\mathrm{Ca}, 1.3 \mathrm{~g} ; \mathrm{P}, 0.2 \mathrm{~g}$; $\mathrm{Na}, 1.57 \mathrm{~g} ; \mathrm{Mg}, 0.1 \mathrm{~g} ; \mathrm{Fe}, 18 \mathrm{mg} ; \mathrm{Zn}, 62 \mathrm{mg} ; \mathrm{Mn}, 18 \mathrm{mg}$; Cu, $3.6 \mathrm{mg}$; iodate, $0.39 \mathrm{mg}$; Se, $0.34 \mathrm{mg}$; all-trans-retinol, $0.72 \mathrm{mg}$; cholecalciferol, $27.4 \mu \mathrm{g}$; menadione sodium bisulphate, $1.07 \mathrm{mg}$, all-rac- $\alpha$ tocopheryl acetate, $18 \mathrm{mg}$; thiamine- $\mathrm{HCl}, 3 \mathrm{mg}$; riboflavin, $2.7 \mathrm{mg}$; pyridoxine- $\mathrm{HCl}, 4.9 \mathrm{mg}$; folic acid, $1.2 \mathrm{mg}$; nicotinic acid, $11 \mathrm{mg}$; pantothenic acid, $8 \mathrm{mg}$; biotin, $0.22 \mathrm{mg}$; cyanocobalamine, $0.056 \mathrm{mg}$; choline, $60 \mathrm{mg}$.

care and handling of laboratory animals ${ }^{(24)}$. The animal experiment included a total of sixty male, 3-week-old NMRI mice (Harlan Winkelmann). The NMRI mouse is a general-purpose model used in different fields, including toxicology, teratology, pharmacology and physiology, and therefore suitable to study the effects of long-term carnitine supplementation. Mice were housed in groups of five each under controlled conditions (Makrolon cages, lighting from 07.00 to 19.00 hours, $22 \pm 1^{\circ} \mathrm{C}$ ambient temperature, 50-60\% relative humidity). The mice were randomly assigned to two groups (control and carnitine) of thirty mice per group with similar BW in the control group $(12.0(\mathrm{sD} 0 \cdot 2) \mathrm{g})$ and the carnitine group (12.0 (SD 0.3) g). Mice of the two groups were fed the same basal natural ingredient diet containing all nutrients in accordance with National Research Council recommendations of laboratory rodents ${ }^{(25)}$. The composition of the basal natural ingredient diet is presented in Table 1. Soyabean extraction meal was used instead of casein due to its low native carnitine concentration. In the control group, the basal natural ingredient diet, which had a low carnitine concentration of $<5 \mathrm{mg} / \mathrm{kg}$ diet as determined by tandem MS, was fed unsupplemented. In the carnitine group, the basal natural ingredient diet was supplemented with $1000 \mathrm{mg}$ L-carnitine (Carnifeed; Lonza) per kg of diet. The mice had free access to the diets, and food intake was recorded weekly. Water was available ad libitum from nipple drinkers during the whole experiment. All animals were assessed visually each day for normal appearance, behaviour and the absence of any signs of impaired health. No adverse events in any of the mice were observed during the whole experiment.

\section{Sample collection}

At an age of 10, 15 and 19 months, ten mice of each group were decapitated under $\mathrm{CO}_{2}$ anaesthesia. Blood was collected into EDTA polyethylene tubes (Sarstedt), and plasma was separated from blood by centrifugation at $1100 \boldsymbol{g}$ at $4^{\circ} \mathrm{C}$ for $\left.10 \mathrm{~min}\right)$. The liver and an aliquot of skeletal muscle (Musculus gastrocnemius) were excised and immediately snap-frozen in liquid $\mathrm{N}_{2}$. Afterwards, all samples were stored at $-80^{\circ} \mathrm{C}$ pending analysis. 
Analysis of carnitine and its precursors in plasma and tissues

Concentrations of free carnitine, acetylcarnitine, $\boldsymbol{\gamma}$-butyrobetaine $(\gamma$-BB) and trimethyllysine in plasma, liver and M. gastrocnemius were determined using tandem MS according to Hirche et al. ${ }^{(26)}$.

\section{Analysis of TAG and cholesterol in plasma and liver}

Lipids from liver samples were extracted with a mixture of $n$-hexane and isopropanol (3:2, v/v) according to Hara \& $\operatorname{Radin}^{(27)}$. Lipid extracts were dried and lipids dissolved with chloroform and Triton X-100 (1:1, v/v) as described by Eder \& Kirchgessner ${ }^{(28)}$. TAG and cholesterol contents were measured using enzymatic reagent kits (Fluitest CHOL, catalogue no. 4241; Fluitest TG, catalogue no. 5741; both from Analyticon Biotechnologies).

\section{Total RNA isolation from liver and skeletal muscle}

Total RNA from $10 \mathrm{mg}$ frozen liver aliquots was isolated using TRIzol reagent (Invitrogen) according to the manufacturer's protocol. Total RNA from $30 \mathrm{mg}$ frozen skeletal muscle aliquots was extracted using RNeasy Mini Kit (Qiagen) according to the manufacturer's protocol. Afterwards, total RNA concentration and purity were estimated from the optical density at 260 and $280 \mathrm{~nm}$, respectively, using an Infinite 200M microplate reader equipped with a NanoQuant plate (both from Tecan). The average optical density A260:A280 ratio of all liver total RNA samples was 1.94 (SD 0.01, $n$ 10) and 1.92 (SD 0.05, $n$ 10) for the control group and the carnitine group, respectively. The average optical density A260:A280 ratio of all skeletal muscle total RNA samples was 1.87 (SD 0.02, $n$ 10) and 1.87 (sD $0.02, n 10)$ for the control group and the carnitine group, respectively.

\section{Quantitative real-time $P C R$ analysis}

Synthesis of cDNA and qPCR analysis on a Rotor-Gene Q system (Qiagen) were carried out as described recently in detail $^{(29)}$. Briefly, for qPCR the KAPA SYBR FAST qPCR Mastermix from Peqlab and gene-specific primer pairs from Eurofins MWG Operon were used. Characteristics of primers used for qPCR analysis of hepatic gene expression and for qPCR validation of microarray data in skeletal muscle are shown in online Supplementary Tables S1 and S2, respectively. The qPCR protocol comprised an initial heating step at $95^{\circ} \mathrm{C}$ for 3 min followed by $35-40$ cycles of a three-step reaction $\left(95^{\circ} \mathrm{C}\right.$ for $3 \mathrm{~s}, 60^{\circ} \mathrm{C}$ for $20 \mathrm{~s}, 72^{\circ} \mathrm{C}$ for $1 \mathrm{~s}$ ). The reaction was followed by a melting curve from 50 to $95^{\circ} \mathrm{C}$ in $5 \mathrm{~s}$ increments of $1^{\circ} \mathrm{C}$ to ensure amplification specificity. All PCR products were separated electrophoretically using a $1.5 \%$ agarose gel stained with GelRed ${ }^{\mathrm{TM}}$ nucleic acid gel stain (Biotium) to confirm the amplification of a single PCR product of the expected size. For calculation of mRNA levels Ct-values of reference and target genes were obtained using Rotor-Gene Q Software (Qiagen). Raw Ct-values of reference genes were transformed into relative expression values using the $2^{-\Delta C t}$ equation for the calculation of normalisation factors. The highest relative value of each gene was set to (1). From these values, the normalisation factor was calculated as the geometric mean of expression data of the three most stable (liver: Actb, Atp $5 b$, Rpl13a; skeletal muscle: Eif4a2, Actb, Rpl13a) out of six potential reference genes according to Vandesompele et al. ${ }^{(30)}$. The stability $M$-values of the three most stable reference genes in liver and skeletal muscle were $<0.55$ and $<0.70$, respectively. Ct-values of target genes were also transformed into relative expression values using the $2^{-\Delta \mathrm{Ct}}$ equation and were normalised with the individual normalisation factor resulting in relative gene quantities that were used for the statistical analysis.

\section{Immunoblotting}

Immunoblotting was carried out as described recently in detail $^{(31)}$. In brief, homogenates were prepared from frozen liver aliquots using radioimmunoprecipitation assay buffer. From each homogenate, the same amount of protein $(30 \mu \mathrm{g})$ was separated on $12.5 \%$ SDS-PAGE and electrotransferred to a nitrocellulose membrane. After incubating the membranes overnight at $4^{\circ} \mathrm{C}$ in blocking solution, membranes were incubated with primary antibodies against ALDH9A1 (polyclonal; Abnova), BBOX1 (monoclonal; Abcam), OCTN2 (polyclonal; LifeSpan Biosciences), glutathione peroxidase (polyclonal; Abcam), heme oxygenase (monoclonal; Abcam) and $\beta$-actin (monoclonal; Abcam) as a reference protein to control for adequate normalisation at room temperature. Following a washing step, membranes were incubated with a horseradish-peroxidase-conjugated secondary monoclonal anti-mouse-IgG (Sigma-Aldrich) or polyclonal anti-mouse-IgG antibodies (DakoCytomation) at room temperature and subsequently developed using ECL Plus (GE Healthcare). The signal intensities of specific bands were detected with a Bio-Imaging system (Syngene) and quantified using Syngene GeneTools software (non-linear dynamics). For calculation of protein levels, the band intensity of the proteins of interest was normalised by that of $\beta$-actin.

\section{Microarray analysis and bioinformatic analysis of microarray data}

For microarray analysis of skeletal muscle, five skeletal muscle total RNA samples were randomly selected each from the control group and the carnitine group. Prior to RNA sample processing, RNA quality was checked using an Agilent 2100 Bioanalyser (Agilent technologies). RNA integrity number values were $7 \cdot 4(\mathrm{sD} 0 \cdot 7, n 5)$ and $7 \cdot 5$ (sD 0.3, $n 5$ ) for the control group and the carnitine group, respectively. RNA samples were processed at the 'Kompetenzzentrum Fluoreszente Bioanalytik' (Regensburg) using Affymetrix GeneChip Mouse Gene 2.0 Sense Target arrays as described ${ }^{(32)}$. The microarray data have been submitted in MIAME compliant format to the NCBI's Gene Expression Omnibus public repository ${ }^{(33)}$ (GEO accession no. GSE120120). Microarray analysis was carried out using the Robust Multichip Analysis algorithm ${ }^{(34)}$. Transcripts were defined as differentially expressed when the fold change (FC) between carnitine and control group was $>1 \cdot 2$ or $<-1 \cdot 2$, and $P$ value of the unpaired Student's $t$ test was $<0 \cdot 05$. In order to extract biological meaning from the list of differentially expressed genes, gene set enrichment analysis 
Table 2. Body weight (BW) development and food intake of mice fed a control diet or a diet supplemented with $1000 \mathrm{mg} / \mathrm{kg}$ diet L-carnitine from 3 weeks to 19 months of age ( $n 10$ mice/group)

(Mean values and standard deviations)

\begin{tabular}{|c|c|c|c|c|c|}
\hline & \multicolumn{2}{|c|}{ Control } & \multicolumn{2}{|c|}{ Carnitine } & \multirow[b]{2}{*}{$P$} \\
\hline & Mean & SD & Mean & SD & \\
\hline BW at 3 weeks $(\mathrm{g})$ & $12 \cdot 0$ & 0.2 & $12 \cdot 0$ & 0.3 & 0.93 \\
\hline BW at 10 months $(\mathrm{g})$ & $56 \cdot 4$ & 5.4 & 59.8 & 4.9 & 0.16 \\
\hline BW at 15 months $(\mathrm{g})$ & $56 \cdot 2$ & 7.5 & 53.5 & 7.9 & 0.45 \\
\hline BW at 19 months $(\mathrm{g})$ & $57 \cdot 7$ & 4.4 & $56 \cdot 4$ & $4 \cdot 3$ & 0.18 \\
\hline Total BW gain $(\mathrm{g})$ & $45 \cdot 7$ & 4.4 & 44.5 & 4.4 & 0.52 \\
\hline DFI from 3 weeks to 10 months $(\mathrm{g})$ & $6 \cdot 4$ & 0.7 & $6 \cdot 3$ & 0.5 & 0.98 \\
\hline DFI from 10 to 15 months $(\mathrm{g})$ & $6 \cdot 0$ & 0.7 & $6 \cdot 2$ & 0.4 & 0.69 \\
\hline DFI from 15 to 19 months $(\mathrm{g})$ & 5.5 & 0.6 & $5 \cdot 8$ & 0.6 & 0.58 \\
\hline
\end{tabular}

$\mathrm{DFI}$, daily food intake.

(GSEA) was performed separately for the up- and downregulated genes using Gene Ontology (GO) biological process and GO molecular function terms using the Database for Annotation, Visualisation and Integrated Discovery 6.8 bioinformatic resource ${ }^{(35,36)}$.

\section{Statistical analysis}

Data are shown as means and standard deviations. The data were subjected to ANOVA using the Minitab Statistical Software Rel. 13.1 (Minitab). Data considering different ages of the mice (carnitine metabolites and lipids in plasma and tissues) were subjected to two-factorial ANOVA with classification factors being carnitine (Carn), age (Age) and the interaction of both factors (Carn $\times$ Age). For statistically significant $F$ values, individual means of the treatment groups were compared by Fisher's multiple range test. Corresponding non-parametric tests were used to analyse data with non-normal distributions. Data considering only one age of the mice (transcript measurements, immunoblotting) were analysed by Student's $t$ test. Means were considered significantly different for $P<0 \cdot 05$.

The number of mice per group for detecting a significant difference among groups (main parameter: plasma-free carnitine) was calculated by G*Power (rel. 3.1.2) assuming a hypothesised effect size of 1.35 , a type I error of 0.05 (two sided) and a statistical power of $0 \cdot 80$.

\section{Results}

Daily food intake, body weight of the mice at 10, 15 and 19 months of age and total body weight gain

Daily food intake during the experiment, BW at 10, 15 and 19 months of age and total BW gain from 3 weeks to 19 months of age did not differ between the control and the carnitine group (Table 2).

\section{Concentrations of carnitine and its precursors in plasma} and tissues of the mice at 10, 15 and 19 months of age

The concentrations of free carnitine, acetylcarnitine and the sum of free carnitine and acetylcarnitine in plasma, liver and skeletal muscle were higher in mice of the carnitine group than in those of the control group $(P<0 \cdot 05$, Fig. 1(A)-(C)). Plasma concentration of free carnitine and the sum of free carnitine and acetylcarnitine were higher in mice at adult age (10 and 15 months) than in mice at advanced age (19 months) $(P<0.05)$. While the concentrations of free carnitine and the sum of free carnitine and acetylcarnitine in liver and skeletal muscle were not influenced by the age of the mice, the concentration of acetylcarnitine in liver and muscle was influenced by the age of the mice. Muscle acetylcarnitine concentration was decreased in mice at advanced age (19 months) compared with mice at adult age (10 and 15 months) $(P<0 \cdot 05)$. Hepatic acetylcarnitine concentration, which made up less than $2 \%$ of the sum of free carnitine and acetylcarnitine, was increased in mice at advanced age (19 months) compared with mice at adult age (10 and 15 months) $(P<0 \cdot 05)$.

The concentrations of $\gamma$-BB in plasma, liver and skeletal muscle were 4-5-, 4-5- and 2-3-fold higher in mice of the carnitine group than in those of the control group $(P<0 \cdot 05$, Fig. 2(A)-(C)). The age of the mice had no influence on the concentrations of $\gamma$-BB in all tissues investigated. Plasma concentration of trimethyllysine was $15-20 \%$ higher in mice of the carnitine group than in those of the control group $(P<0.05$, Fig. 2(A)) but was not influenced by the age of the mice.

\section{Concentrations of TAG and cholesterol in plasma and liver of the mice at 10 and 19 months of age}

The concentrations of TAG and cholesterol in plasma and liver did not differ between mice of the carnitine group and those of the control group at 10 and 19 months of age (online Supplementary Fig. S1).

\section{Relative mRNA and protein levels of key genes involved in lipid metabolism in the liver of mice at 19 months of age}

Relative mRNA concentrations of PPAR $\alpha$-regulated genes involved in lipid catabolism in the liver of mice at an age of 19 months either did not differ between the two groups (acylCoA dehydrogenase medium chain ( $\mathrm{Acadm}$ ), solute carrier family 25 member 20/carnitine/acylcarnitine translocase (Slc25a20/Cact), solute carrier family 27 member $1 /$ fatty acid transporter 1 (Slc27a1/Fatp1) and solute carrier family 22 member 5/organic cation transporter 2 (Slc22a5/Octn2)) or were significantly higher (aldehyde dehydrogenase 9 family member A1 (Aldb9a1)) or tended to be higher ( $\gamma$-butyrobetaine hydroxylase 1 (Bbox1)) in the carnitine group than in the control group (online Supplementary Table S3). The relative mRNA concentrations of key genes involved in lipid synthesis (acetyl-CoA carboxylase $\alpha$ (Acaca), fatty acid synthase (Fasn), glycerol-3-phosphate acyltransferase, mitochondrial (Gpam) and stearoyl-CoA desaturase ( $S c d 1)$ ), which are under the control of sterol regulatory element-binding proteins (SREBP), in the liver of mice at an age of 19 months did not differ between the carnitine group and the control group (online Supplementary Table S3). Relative protein concentrations of ALDH9A1, BBOX1 and OCTN2, which are involved in the regulation of carnitine homeostasis, in the liver of mice at an age of 19 months were not different between the carnitine group and the control group (online Supplementary Fig. S2). 
(A)

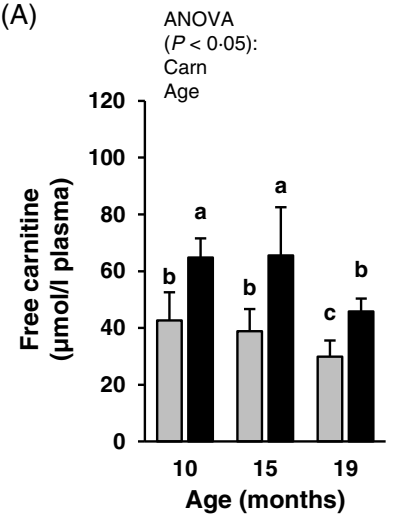

(B)



(C)



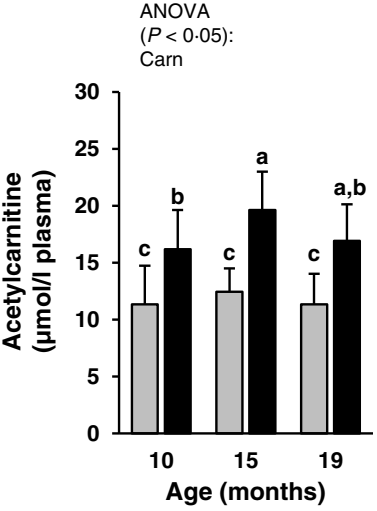
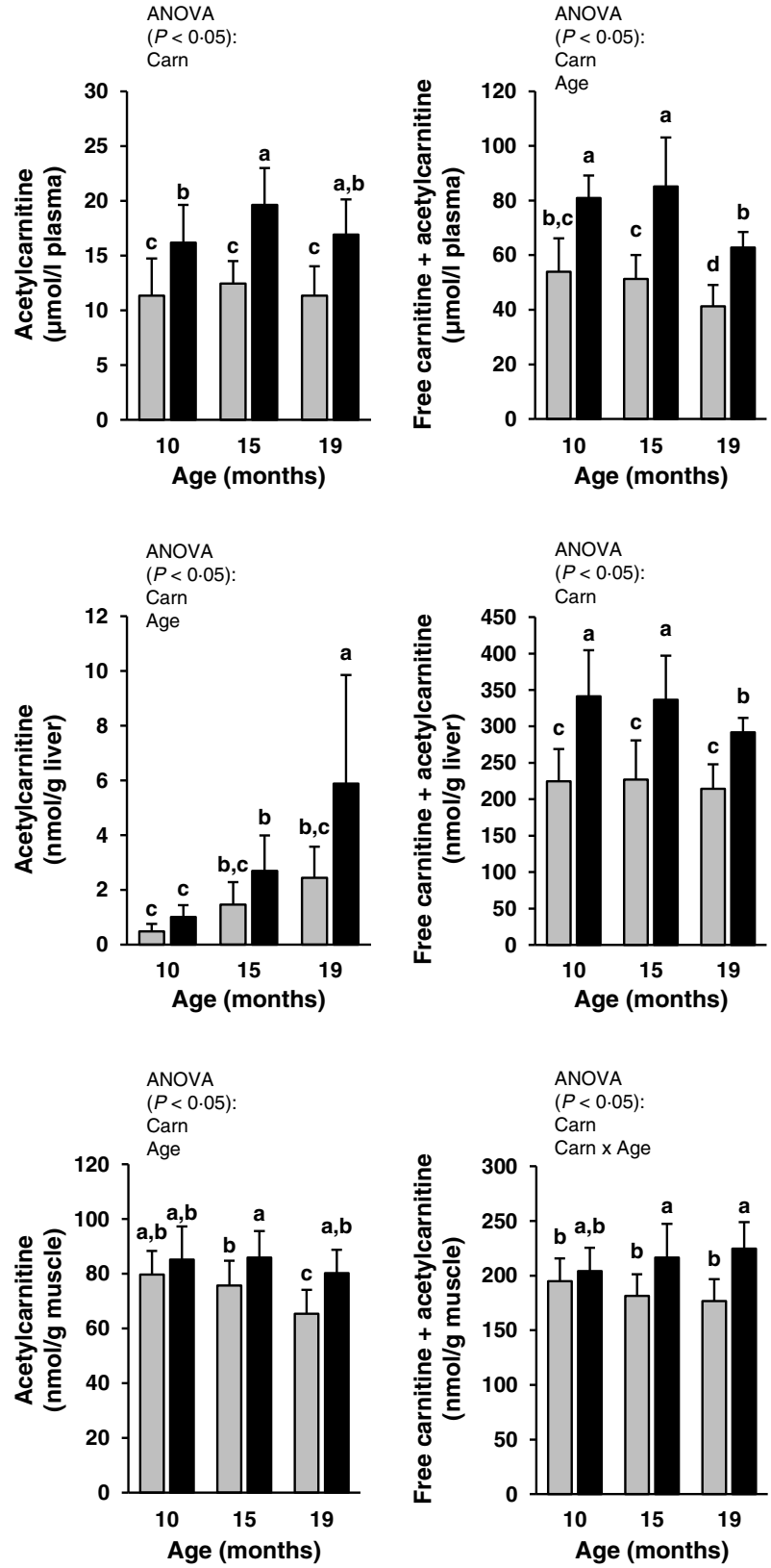

Fig. 1. Concentrations of free carnitine, acetylcarnitine and the sum of free carnitine and acetylcarnitine in plasma (A), liver (B) and skeletal muscle (C) of mice at 10,15 and 19 months of age fed a control diet ( ) or a diet supplemented with $1000 \mathrm{mg} / \mathrm{kg}$ diet L-carnitine (-) from 3 weeks to 19 months of age. Bars represent mean values and standard deviations for $n 10$ mice/group. a,b,c,d Mean values with unlike letters are significantly different $(P<0.05)$.

\section{Relative mRNA and protein levels of genes involved in} stress signalling in the liver of mice at 19 months of age

Relative mRNA concentrations of genes regulated by important stress signalling pathways (ER stress/unfolded protein response, $\mathrm{NF}-\kappa \mathrm{B}$, nuclear factor-E2 related factor 2 ) in the liver of mice at an age of 19 months were not different between the carnitine group and the control group (online Supplementary Table S4). Relative protein concentrations of glutathione peroxidase and heme oxygenase, which are regulated by NF- $\kappa \mathrm{B}$ and nuclear factorE2 related factor 2, respectively, in the liver of mice at an age of 19 months did not differ between the carnitine group and the control group (online Supplementary Fig. S3).

\section{Identification of genes regulated by carnitine supplementation in skeletal muscle of mice at 19 months of age}

A total of 447 genes with official gene symbols ( 171 with $\mathrm{FC}>1 \cdot 2$, 276 with $\mathrm{FC}<-1 \cdot 2, P<0 \cdot 05)$ were identified as differentially expressed in skeletal muscle of 19-month-old mice between the carnitine and the control group (online Supplementary Tables S5 and S6). The most strongly up- and down-regulated genes in the carnitine group compared with the control group were $\beta-1,4-N$-acetyl-galactosaminyl transferase 2 (B4galnt2) $(+3 \cdot 11$-fold) and the ankyrin repeat domain 2 (Ankrd2) $(-2 \cdot 52$-fold), respectively. Validation of microarray data by 

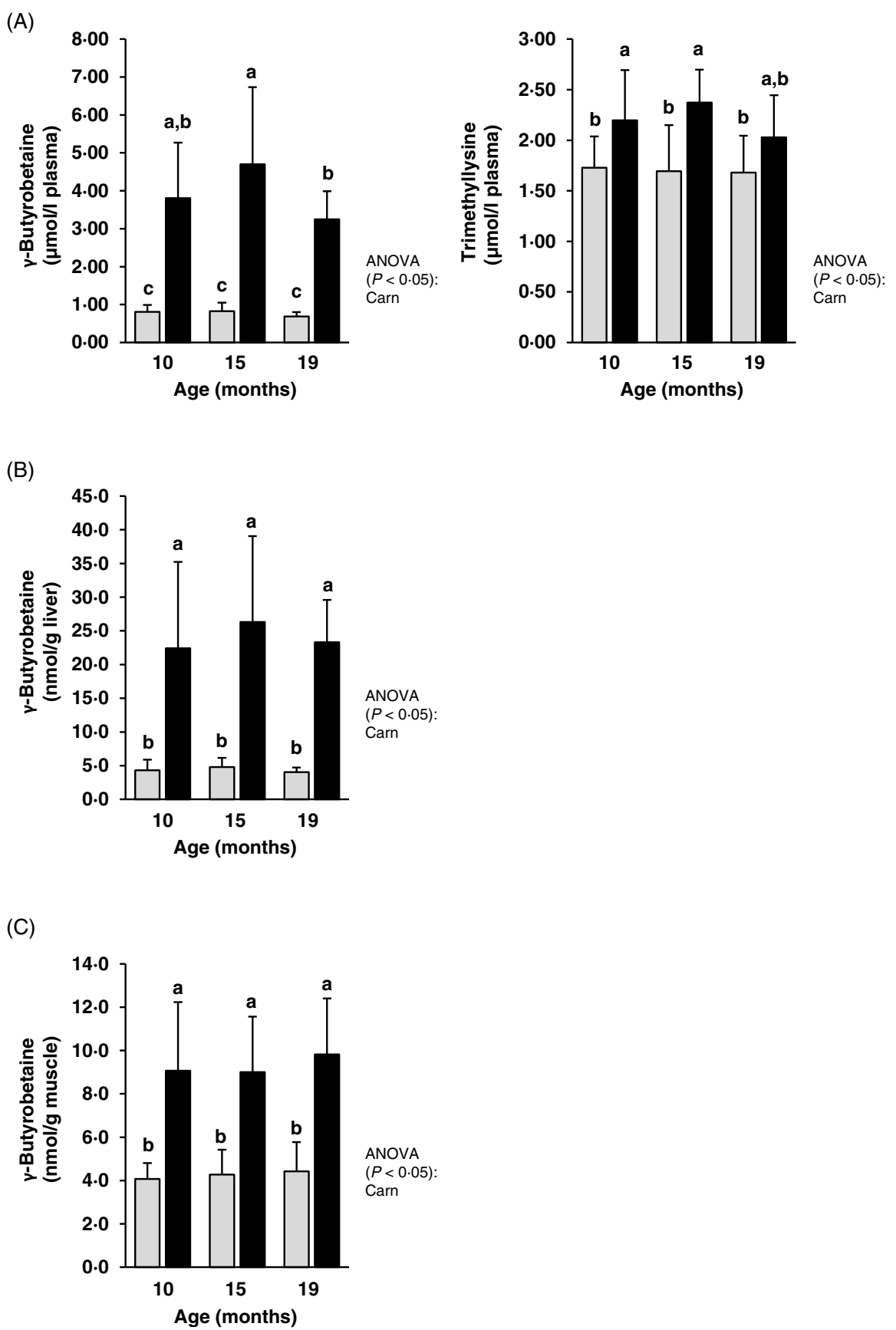

Fig. 2. Concentration of the carnitine precursor $\gamma$-butyrobetaine and trimethyllysine in plasma (A), liver (B) and skeletal muscle (C) of mice at 10,15 and 19 months of age fed a control diet ( ) or a diet supplemented with $1000 \mathrm{mg} / \mathrm{kg}$ diet L-carnitine ( $\mathbf{\bullet}$ ) from 3 weeks to 19 months of age. Bars represent mean values and standard deviations for $n 10$ mice/group. ${ }^{\text {a,b,c }}$ Mean values with unlike letters are significantly different $(P<0.05)$.

qPCR analyses of twelve differentially expressed genes showed that for all genes validated effect direction was the same between qPCR and microarray, but effect size (FC) differed more or less between qPCR and microarray (Table 3). Due to the different number of biological replicates (microarray: $n$ 5, qPCR: $n$ 10) and the different detection principle of transcript abundance between microarray and qPCR the FC and $P$ values differed more or less. Statistical analysis revealed that five out of the twelve mRNA analysed by qPCR were regulated by carnitine supplementation at a significance level of $P<0 \cdot 05$.

\section{Identification of biological processes and molecular functions affected by carnitine supplementation in skeletal muscle of mice at 19 months of age}

To identify biological processes and molecular functions affected by the genes regulated by long-term supplementation of carnitine in skeletal muscle of mice, GSEA was performed using GO biological process and molecular function terms. GSEA of the 171 up-regulated genes revealed that the topenriched GO biological process terms (lowest enrichment 
Table 3. Quantitative real-time PCR (qPCR) validation of microarray data for selected differentially expressed transcripts (fold change $(F C)>1.2$ or $<-1.2, P<0.05)$ in skeletal muscle of 19-month-old mice fed a control diet or a diet supplemented with $1000 \mathrm{mg} / \mathrm{kg}$ diet L-carnitine from 3 weeks to 19 months of age*

\begin{tabular}{lccc}
\hline & \multicolumn{2}{c}{ FC carnitine $v$. control } & $P$ \\
\cline { 2 - 3 } Gene symbol & Microarray & qPCR & qPCR \\
\hline B4galnt2 & 3.11 & 2.20 & 0.155 \\
Perp & 2.16 & 1.92 & 0.032 \\
Pld5 & 2.16 & 2.41 & 0.027 \\
Ostn & 2.09 & 1.51 & 0.113 \\
Gamt & 1.53 & 2.66 & 0.049 \\
Tppp3 & 1.48 & 1.75 & 0.014 \\
Ankrd2 & -2.52 & -2.08 & 0.036 \\
Olfr142 & -1.53 & -1.20 & 0.470 \\
Olfr618 & -1.46 & -1.14 & 0.669 \\
Olfr1052 & -1.43 & -1.33 & 0.666 \\
Olfr1356 & -1.40 & -1.28 & 0.520 \\
Olfr427 & -1.38 & -1.23 & 0.612 \\
\hline B4g15 & &
\end{tabular}

B4galnt2, $\beta$-1,4- $N$-acetyl-galactosaminyltransferase 2; Perp, PERP, TP53 apoptosis effector; PId5, phospholipase D family member 5; Ostn, osteocrin; Gamt, guanidinoacetate $\mathrm{N}$-methyltransferase; Tppp3, tubulin polymerisation promoting protein family member 3; Ankrd2, ankyrin repeat domain 2; Olfr, olfactory receptor.

* The microarray FC was calculated from the signal log ratios as follows: $2^{\text {Signallog ratio }}$ if signal $\log$ ratio $\geq 0$ and $(-1) \times 2^{- \text {(Signal log ratio) }}$, if signal log ratio $<(0)$. Signal log ratios were calculated from five microarrays/group. The qPCR FC was calculated analogously from normalised $2^{-\Delta C t}$ ratios. Normalised 2- ${ }^{\Delta C t}$ expression was calculated from $n 10$ mice/group.

$P$ value) were macromolecular complex assembly, homotypic cell-cell adhesion and cellular component biogenesis. The top-enriched GO molecular function terms (lowest enrichment $P$ value) of the 171 up-regulated genes were peptidase activity, ubiquitin-like protein-specific protease activity and enzyme binding. All GO biological process and molecular function terms assigned to the up-regulated genes with an enrichment $P$ value $<0.01$ are shown in online Supplementary Table S7.

GSEA of the 276 down-regulated genes showed that the topenriched biological process terms (enrichment $P$ value $<0 \cdot 01$ ) were in decreasing order sensory perception of smell, sensory perception of chemical stimulus and sensory perception. The most enriched GO molecular function terms (enrichment $P$ value $<0 \cdot 01$ ) were in decreasing order G-protein-coupled receptor activity, olfactory receptor activity and transmembrane receptor activity. All GO biological process and molecular function terms assigned to the down-regulated genes with an enrichment $P$ value $<0 \cdot 01$ are shown in online Supplementary Table S8.

\section{Discussion}

An important finding of the present study is that lifelong carnitine supplementation at a dose comparable with that used in clinical trials is effective in preventing the age-dependent impairment of plasma carnitine status. In addition, a key finding of the present study is that feeding of supplemental carnitine during whole life generally leads to an elevated carnitine status in plasma and tissues of mice up to advanced age compared with age-matched non-supplemented control mice. This clearly indicates that the animal's plasma and tissue carnitine status profits from lifelong carnitine supplementation. With regard to the effect of carnitine supplementation on carnitine status, some tissue specificities were found, such as supplemental dietary carnitine increased the concentration of the sum of free carnitine and acetylcarnitine in plasma and liver markedly stronger than in skeletal muscle. In addition, skeletal muscle carnitine status was improved by carnitine supplementation only at an age of 15 months and above. This relative 'resistance' of skeletal muscle carnitine content to even high doses of supplemental dietary carnitine is in agreement with findings from other studies in mice ${ }^{(37)}$, $\operatorname{rats}^{(38,39)}$ and humans ${ }^{(40,41)}$ and has been attributed to the specific characteristics of skeletal muscle carnitine uptake which is mediated by OCTN2-transporting carnitine in a $\mathrm{Na}^{+}$-dependent and saturable manner from plasma into skeletal muscle against a considerable concentration gradient $(>100 \text {-fold })^{(42)}$. Due to the $K_{m}$ of OCTN2 for carnitine $\left(4.3 \mu \mathrm{M}^{(43)}\right)$, muscle OCTN2 is saturated even under normal blood levels of carnitine, and it is unlikely that increasing plasma carnitine availability increases muscle carnitine content. However, muscle carnitine content can be increased by $15-20 \%$ by experimentally induced hypercarnitinaemia combined with intravenously infusing insulin or ingesting relatively large quantities of carbohydrates ${ }^{(44,45)}$. The stimulatory effect of insulin on skeletal muscle carnitine uptake has been explained by increasing sarcolemmal $\mathrm{Na}^{+} / \mathrm{K}^{+}$-ATPase activity and intracellular $\mathrm{Na}^{+}$flux, thereby increasing $\mathrm{Na}^{+}$-carnitine cotransport via OCTN2 ${ }^{(46)}$. Nonetheless, species differences exist with regard to the effect of exogenous carnitine on muscle carnitine content. In pigs, feeding the same dose of supplemental carnitine as in the present study for only 3-4 weeks increased the concentration of free carnitine and acetylcarnitine in skeletal muscle by greater than $2 \cdot 5-$ fold $^{(47,48)}$. Although the exact reason for these species differences is unknown, it could be speculated that pig muscle has a higher capacity for carnitine uptake from plasma due to higher expression level of Octn2 in skeletal muscle than in rodents and humans.

As a reason for the impaired plasma carnitine status of mice at advanced age, a decreased intestinal absorption and renal reabsorption of carnitine might come into question. Both intestinal absorption of dietary carnitine and renal reabsorption of filtered carnitine are dependent on the activity of the aforementioned OCTN2 present in intestinal epithelial cells ${ }^{(49)}$ and renal tubular cells ${ }^{(50)}$. Interestingly, a recent study clearly showed that $\mathrm{Na}^{+}$-dependent carnitine uptake in renal brush-border membrane vesicles isolated from whole kidney is significantly decreased in 8-month-old rats compared with 2-month-old rats $^{(51)}$. In another study, it was demonstrated that mRNA expression of Octn2 in jejunum and ileum is lower in 6 -month-old rats than in 1 -month-old rats ${ }^{(52)}$. In addition, in humans a down-regulation of Octn 2 was observed in mononuclear cells of healthy elderly compared with healthy adults ${ }^{(53)}$. These findings from rats and humans are indicative of an age-induced impairment of $\mathrm{Na}^{+}$-dependent carnitine uptake. Despite direct proof in mice is lacking, an age-induced decline of intestinal carnitine absorption and renal carnitine reabsorption may therefore account for the decreased plasma carnitine status in mice at advanced age. Apart from intestinal absorption and renal reabsorption, plasma carnitine status is influenced by endogenous synthesis of carnitine in certain tissues. Endogenous synthesis of carnitine occurs via stepwise, 
enzyme-catalysed conversion of free trimethyllysine, the initial carnitine precursor, into $\gamma-\mathrm{BB}$, the ultimate precursor of carnitine, which is finally hydroxylated by $\gamma$-BB hydroxylase 1 (encoded by Bbox1) into carnitine. Because the activity of $\gamma$-BB hydroxylase 1 is restricted to liver and kidney in most mammals ${ }^{(54)}$, these two organs play a central role in endogenous carnitine biosynthesis. However, non-hepatic and non-renal tissues, like skeletal muscles, also contribute to carnitine biosynthesis through providing $\gamma$-BB. In the present study, the plasma concentration of free trimethyllysine, whose availability is considered to be limiting for carnitine biosynthesis $^{(55)}$, and plasma and tissue concentrations of $\gamma$-BB in control mice were not different between the different age groups. This indicates that carnitine biosynthesis is probably not impaired at advanced age compared with adult age. In the context of $\gamma$-BB, a further interesting observation was that its plasma and tissue concentration was generally markedly higher in carnitine-supplemented mice than in control mice. Elevated plasma levels of $\boldsymbol{\gamma}$-BB in carnitine-supplemented subjects have been also reported from others ${ }^{(56,57)}$ and have been suggested to be the result of inhibition of $\gamma$-BB hydroxylase 1 activity by carnitine ${ }^{(58)}$, thereby resulting in the accumulation of $\gamma$-BB. However, it has also been shown that gut microbial metabolism of dietary carnitine produces quantitatively significant amounts of $\gamma$-BB in the gut, particularly in the small intestine, of mice ${ }^{(59)}$ and rats ${ }^{(60,61)}$, and $\gamma$-BB is a good substrate for the carnitine transporter OCTN2 ${ }^{(43)}$, thereby facilitating the intestinal absorption of $\gamma$-BB. In line with this, Koeth et al. ${ }^{(59)}$ demonstrated that plasma levels of $\gamma$-BB in carnitinesupplemented mice showed a 100-fold increase compared with control mice receiving no carnitine. Furthermore, suppression of gut microbiota with an oral antibiotic cocktail eliminated plasma levels of $\gamma$-BB in carnitine-supplemented mice ${ }^{(59)}$, which strongly suggests that the majority of plasma $\gamma$-BB in carnitine-supplemented mice originates from gut microbial metabolism of dietary carnitine and not from inhibition of enzymes involved in carnitine synthesis.

In order to investigate the implications of carnitine supplementation-induced improvement of carnitine status in the liver of mice at advanced age, we studied the effect on key metabolic and stress signalling pathways in the liver by determining mRNA and protein levels of genes involved in these pathways. Hepatic mRNA levels of genes involved in lipid synthesis, which are under the control of SREBP, and of most genes involved in lipid catabolism, which are regulated by PPAR $\alpha$, were not different between 19-month-old mice of the control group and the carnitine group. An exception was that the hepatic mRNA level of Aldh9a1 was elevated, and that of Bbox 1 tended to be elevated in the carnitine group compared with the control group. Despite being speculative, it might be that increased mRNA levels of genes involved in carnitine biosynthesis (Aldh9a1 and Bbox1) is a compensatory effect to the known inhibition of $\boldsymbol{\gamma}$-BB dioxygenase activity by carnitine ${ }^{(58)}$. However, at the protein level, no increase in the expression of ALDH9A1, BBOX1 and OCTN2 was seen in the liver of the carnitine group. Thus, taken together, we suggest that key lipid metabolic pathways regulated by SREBP and PPAR $\alpha$ in the liver are not affected by carnitine supplementation in mice of advanced age. In line with this, the concentrations of TAG and cholesterol in liver and plasma did not differ between mice of the two groups at 19 months of age. This clearly indicated that lifelong carnitine supplementation has neither beneficial nor detrimental effects on hepatic lipid metabolism. Apart from key metabolic pathways in the liver, we also considered possible implications of the improvement of carnitine status in the liver of carnitine-supplemented mice at advanced age on hepatic stress signalling and stress adaptation pathways. Our investigations at the mRNA and protein level revealed no effect of carnitine supplementation on expression of genes involved in important stress signalling and stress adaptation pathways in the liver of 19-month-old mice between the control and the carnitine group. Genes involved in ER stress/unfolded protein response signalling and genes regulated by NF- $\mathrm{KB}$ and nuclear factor-E2 related factor 2 , which are known to be induced in response to ER stress-induced unfolded protein response in order to improve cellular protective mechanisms to cope with ER stress ${ }^{(62)}$, are very sensitive to different kinds of stressors including metabolic, inflammatory, prooxidative and microbial signals $^{(63)}$. Despite acute ER stress induces several homeostatic mechanisms to preserve ER function and cell function, chronic induction of ER stress has been shown to play a pathophysiological role in a number of diseases, such as fatty liver, obesity, diabetes and many others ${ }^{(64)}$. Thus, the unaltered expression of ER stress-sensitive genes in the liver of 19month-old mice suggests that lifelong carnitine supplementation does not represent a stressful condition to the animal.

Apart from the liver, we also studied possible implications of carnitine supplementation-induced improvement of carnitine status in skeletal muscle of mice at advanced age by genomewide transcript profiling. Despite the large number of genes identified as differentially regulated by supplemental carnitine, which is partially attributed to the mild filter settings applied, the very low number of genes regulated more strongly (e.g. only ten and six genes were regulated $>1.5$-fold and $<-1.5$-fold, respectively) indicates that the influence of lifelong carnitine supplementation on the skeletal muscle transcriptome of 19-month-old mice is very moderate. The most strongly upregulated gene in skeletal muscle of the carnitine group was B4galnt 2 which encodes an acetylgalactosaminyl transferase that is confined in expression to specific specialised membrane domains in skeletal muscle ${ }^{(65)}$. Interestingly, overexpression of B4galnt2 in skeletal muscle was reported to inhibit the development of muscle pathology in a mouse model of congenital form of muscular dystrophy ${ }^{(66)}$. By contrast, the most strongly down-regulated gene in skeletal muscle of the carnitine group was Ankrd2 encoding the most abundant isoform of muscle ankyrin repeat proteins, which are stress-responsive proteins transducing different stress signals such as stretch, denervation and exercise in skeletal muscle ${ }^{(67-69)}$. Although the true biological meaning of carnitine-induced regulation B4galnt2 and Ankrd2 in skeletal muscle of mice is hard to interpret, it indicates that dietary carnitine has the potential to modulate pathophysiological processes and stress responses in skeletal muscle. To further interpret the biological meaning of carnitine supplementation-induced changes in the skeletal muscle transcriptome of 19-month-old mice, GSEA was performed with 
the differentially regulated genes. Noteworthy, GSEA for the down-regulated genes showed an involvement of the encoded gene products in sensory perception of smell and G-protein coupled receptor signalling pathway. The enrichment of these biological processes is a highly interesting observation which is explained by the fact that the list of down-regulated genes contained a large number of genes ( $>60$ ) encoding olfactory receptors (Olfr isoforms) and vomeronasal 1 and 2 receptors (Vmn1r and Vmn2r isoforms). Receptors of the Olfr, Vmn1r and Vmn2r gene families comprise the largest family of $G$ protein-coupled receptors, which recognise and sense volatile chemicals (i.e. odorants) serving as exogenous ligands in order to facilitate perception of smell in the brain ${ }^{(70)}$. Due to this, these receptors are extensively expressed in the nasal olfactory epithelium, specifically in the cilia of olfactory sensory neurons. However, wholegenome sequencing studies convincingly demonstrated that these receptors are also ectopically expressed in non-chemosensory tissues like testis, kidney and skeletal muscle ${ }^{(71)}$, where these receptors are also functional and activated by endogenous ligands. Interestingly, recent evidence indicates that skeletal muscle Olfr isoforms play a role in skeletal muscle development, regeneration and even muscular dystrophy ${ }^{(72,73)}$. Thus, although the biological significance of the results from GSEA should also be interpreted cautiously considering the overall very moderate regulation of genes by carnitine supplementation in mice, the regulation of more than sixty genes encoding chemosensory receptors by carnitine supplementation in skeletal muscle of 19-month-old mice strongly suggests that supplemental carnitine has the potential to modulate processes involved in muscle physiology and pathophysiology. Future studies are warranted to clarify the biological consequences of regulation of a large set of genes encoding chemosensory receptors in skeletal muscle by dietary carnitine.

The absence of detrimental effects of carnitine supplementation in our study is in strong contrast to two recent studies in mice demonstrating an impairment of cardiovascular health and liver function by carnitine supplementation ${ }^{(20,21)}$. However, the findings from the two mice studies are also in contrast to the vast majority of clinical studies which did not observe any adverse effects of carnitine supplementation ${ }^{(8,22,23)}$. An important difference between the studies of Koeth et $a l^{(20)}$ and Lu et al. ${ }^{(21)}$ and clinical studies is the extremely high dose of carnitine per $\mathrm{kg}$ BW which was approximately 40-100 times higher in the mice studies than in clinical studies. Considering that in our study, the almost lifelong administration of carnitine at a dose which was similar to that used in clinical studies did not provoke adverse health effects, we postulate that the adverse effects reported recently are the result of the high dose of carnitine administered. It is likely that excessive formation of trimethylamine- $N$-oxide through gut microbiotadependent conversion of carnitine into trimethylamine, which is subsequently converted to trimethylamine- $N$-oxide by hepatic flavin monooxygenase, is responsible for the adverse effects of supplemental carnitine, because multiple detrimental effects of trimethylamine- $N$-oxide have been identified such as inhibition of reverse cholesterol transport and impairment of hepatic and intestinal and sterol metabolism ${ }^{(74,75)}$.

In conclusion, lifelong carnitine supplementation in mice prevents an age-dependent impairment of plasma carnitine status and improves the carnitine status of liver and skeletal muscle up to advanced age. While the improvement of tissue carnitine status in mice at advanced age was not accompanied by any detrimental changes in hepatic lipid metabolism and stress signalling pathways, moderate changes in skeletal muscle transcriptome might be indicative of the potential of carnitine to modulate muscle physiological processes. Based on the present observations, safety concerns associated with long-term supplementation of carnitine can be considered as unfounded, at least at doses used in clinical trials and with regard to the parameters investigated. This assumption is supported by the fact that none of the carnitine supplementation studies in humans reported any adverse effects up to daily doses of $6 \mathrm{~g}$ carnitine per $\mathrm{d}$.

\section{Acknowledgements}

U. B. C. was funded by the German Academic Exchange Service (DAAD; funding ID: 50015451).

U. B. C. performed the animal experiment, carried out qPCR and immunoblot analyses and analysed the data; E. M. carried out carnitine and lipid analyses; R. R. performed bioinformatic analysis of transcriptomic data and wrote the paper; K. E. conceived and designed the experiment.

There are no conflicts of interest.

\section{Supplementary material}

For supplementary material/s referred to in this article, please visit https://doi.org/10.1017/S0007114519000709

\section{References}

1. Fritz IB \& McEwen B (1959) Effects of carnitine on fatty-acid oxidation by muscle. Science 129, 334-335.

2. Lysiak W, Lilly K, DiLisa F, et al. (1988) Quantitation of the effect of L-carnitine on the levels of acid-soluble short-chain acyl-CoA and COASH in rat heart and liver mitochondria. J Biol Chem 263, 1151-1156.

3. Uziel G, Garavaglia B \& Di Donato S (1988) Carnitine stimulation of pyruvate dehydrogenase complex (PDHC) in isolated human skeletal muscle mitochondria. Muscle Nerve 11, 720-724.

4. Broderick TL, Quinney HA \& Lopaschuk GD (1992) Carnitine stimulation of glucose oxidation in the fatty acid perfused isolated working rat heart. J Biol Chem 267, 3758-3763.

5. De Palo E, Gatti R, Sicolo N, et al. (1981) Plasma and urine free L-carnitine in human diabetes mellitus. Acta Diabetol Lat 18 , 91-95.

6. Pregant P, Schernthaner G, Legenstein E, et al. (1991) Decreased plasma carnitine in type I diabetes mellitus. Klin Wochenschr 69, 511-516.

7. Poorabbas A, Fallah F, Bagdadchi J, et al. (2007) Determination of free L-carnitine levels in type II diabetic women with and without complications. Eur J Clin Nutr 61, 892-895.

8. Ringseis R, Keller J \& Eder K (2012) Role of carnitine in the regulation of glucose homeostasis and insulin sensitivity: evidence from in vivo and in vitro studies with carnitine supplementation and carnitine deficiency. Eur J Nutr 51, 1-18.

9. Waber LJ, Valle D, Neill C, et al. (1982) Carnitine deficiency presenting as familial cardiomyopathy: a treatable defect in carnitine transport. J Pediatr 101, 700-705. 
10. Glasgow AM, Engel AG, Bier DM, et al. (1983) Hypoglycemia, hepatic dysfunction, muscle weakness, cardiomyopathy, free carnitine deficiency and long-chain acylcarnitine excess responsive to medium chain triglyceride diet. Pediatr Res 17, 319-326.

11. Tripp ME, Katcher ML, Peters HA, et al. (1981) Systemic carnitine deficiency presenting as familial endocardial fibroelastosis: a treatable cardiomyopathy. $N$ Engl J Med 305, 385-390.

12. Gramignano G, Lusso MR, Madeddu C, et al. (2006) Efficacy of L-carnitine administration on fatigue, nutritional status, oxidative stress, and related quality of life in 12 advanced cancer patients undergoing anticancer therapy. Nutrition 22, 136-145.

13. Steiber AL, Davis AT, Spry L, et al. (2006) Carnitine treatment improved quality-of-life measure in a sample of Midwestern hemodialysis patients. JPEN J Parenter Enteral Nutr 30, 10-15.

14. Mantovani G, Macciò A, Madeddu C, et al. (2008) Randomized phase III clinical trial of five different arms of treatment for patients with cancer cachexia: interim results. Nutrition $\mathbf{2 4}$, 305-313.

15. Malaguarnera M, Vacante M, Giordano M, et al. (2011) Oral acetyl-L-carnitine therapy reduces fatigue in overt hepatic encephalopathy: a randomized, double-blind, placebocontrolled study. Am J Clin Nutr 93, 799-808.

16. Rabito EI, Leme IA, Demenice R, et al. (2013) Lower carnitine plasma values from malnutrition cancer patients.J Gastrointest Cancer 44, 362-365.

17. De Simone C, Tzantzoglou S, Jirillo E, et al. (1992) L-Carnitine deficiency in AIDS patients. AIDS 6, 203-205.

18. Vinci E, Rampello E, Zanoli L, et al. (2005) Serum carnitine levels in patients with tumoral cachexia. Eur J Intern Med 16, 419-423.

19. Hockenberry MJ, Hooke MC, Gregurich M, et al. (2009) Carnitine plasma levels and fatigue in children/adolescents receiving cisplatin, ifosfamide, or doxorubicin. J Pediatr Hematol Oncol 31, 664-669.

20. Koeth RA, Wang Z, Levison BS, et al. (2013) Intestinal microbiota metabolism of L-carnitine, a nutrient in red meat, promotes atherosclerosis. Nat Med 19, 576-585.

21. Lu Y, Li W \& Yang X (2017) Soluble soybean polysaccharides enhance the protective effects of genistein against hepatic injury in high L-carnitine-fed mice. Food Funct 8, 4364-4373.

22. Brass EP (2000) Supplemental carnitine and exercise. Am J Clin Nutr 72 Suppl, 618S-623S.

23. Ringseis R, Keller J \& Eder K (2013) Mechanisms underlying the anti-wasting effect of L-carnitine supplementation under pathologic conditions: evidence from experimental and clinical studies. Eur J Nutr 52, 1421-1442.

24. NRC (National Research Council) (2011) Guide for the Care and Use of Laboratory Animals, 8th ed. Washington, DC: National Academies Press.

25. NRC (National Research Council) (1995) Nutrient Requirements of Laboratory Animals, 4th revised ed. Washington, DC: National Academies Press.

26. Hirche F, Fischer M, Keller J, et al. (2009) Determination of carnitine, its short chain acyl esters and metabolic precursors trimethyllysine and gamma-butyrobetaine by quasi-solid phase extraction and MS/MS detection. J Chromatogr B Analyt Technol Biomed Life Sci 877, 2158-2162.

27. Hara A \& Radin NS (1978) Lipid extraction of tissues with a low-toxicity solvent. Anal Biochem 90, 420-426.

28. Eder K \& Kirchgessner M (1994) Dietary fat influences the effect of zinc deficiency on liver lipids and fatty acids in rats force-fed equal quantities of diet. J Nutr 124, 1917-1926.

29. Chiappisi E, Ringseis R, Eder K, et al. (2017) Effect of endoplasmic reticulum stress on metabolic and stress signaling and kidney-specific functions in Madin-Darby bovine kidney cells. J Dairy Sci 100, 6689-6706.
30. Vandesompele J, De Preter K, Pattyn F, et al. (2002) Accurate normalization of real-time quantitative RT-PCR data by geometric averaging of multiple internal control genes. Genome Biol3, RESEARCH0034.

31. Ringseis R, Mooren FC, Keller J, et al. (2011) Regular endurance exercise improves the diminished hepatic carnitine status in mice fed a high-fat diet. Mol Nutr Food Res 55 Suppl. 2, S193-S202.

32. Gessner DK, Winkler A, Koch C, et al. (2017) Analysis of hepatic transcript profile and plasma lipid profile in early lactating dairy cows fed grape seed and grape marc meal extract. BMC Genomics 18, 253.

33. Edgar R, Domrachev M \& Lash AE (2002) Gene Expression Omnibus: NCBI gene expression and hybridization array data repository. Nucleic Acids Res 30, 207-210.

34. Irizarry RA, Hobbs B, Collin F, et al. (2003) Exploration, normalization, and summaries of high density oligonucleotide array probe level data. Biostatistics 4, 249-264.

35. Huang DW, Sherman BT \& Lempicki RA (2009) Bioinformatics enrichment tools: paths toward the comprehensive functional analysis of large gene lists. Nucleic Acids Res 37, 1-13.

36. Huang DW, Sherman BT \& Lempicki RA (2009) Systematic and integrative analysis of large gene lists using DAVID bioinformatics resources. Nat Protoc $\mathbf{4}, 44-57$.

37. Morand R, Bouitbir J, Felser A, et al. (2014) Effect of carnitine, acetyl-, and propionylcarnitine supplementation on the body carnitine pool, skeletal muscle composition, and physical performance in mice. Eur J Nutr 53, 1313-1325.

38. Ringseis R, Lüdi S, Hirche F, et al. (2008) Treatment with pharmacological peroxisome proliferator-activated receptor alpha agonist clofibrate increases intestinal carnitine absorption in rats. Pharmacol Res 58, 58-64.

39. Lambert BD, Dobson CM, Cherry NM, et al. (2009) Chemical form of dietary L-carnitine affects plasma but not tissue carnitine concentrations in male Sprague-Dawley rats. J Anim Physiol Anim Nutr (Berl) 93, 174-180.

40. Barnett C, Costill DL, Vukovich MD, et al. (1994) Effect of L-carnitine supplementation on muscle and blood carnitine content and lactate accumulation during high-intensity sprint cycling. Int J Sport Nutr 4, 280-288.

41. Wächter S, Vogt M, Kreis R, et al. (2002) Long-term administration of L-carnitine to humans: effect on skeletal muscle carnitine content and physical performance. Clin Chim Acta $\mathbf{3 1 8}$, 51-61.

42. Rebouche CJ (1977) Carnitine movement across muscle cell membranes. Studies in isolated rat muscle. Biochim Biophys Acta 471, 145-155.

43. Tamai I, Ohashi R, Nezu J, et al. (1998) Molecular and functional identification of sodium ion-dependent, high affinity human carnitine transporter OCTN(2). J Biol Chem 273, 20378-20382.

44. Stephens FB, Constantin-Teodosiu D, Laithwaite D, et al. (2006) Insulin stimulates L-carnitine accumulation in human skeletal muscle. FASEB J 20, 377-379.

45. Wall BT, Stephens FB, Constantin-Teodosiu D, et al. (2011) Chronic oral ingestion of $\mathrm{L}$-carnitine and carbohydrate increases muscle carnitine content and alters muscle fuel metabolism during exercise in humans. J Physiol 589, 963-973.

46. Stephens FB, Constantin-Teodosiu D \& Greenhaff PL (2007) New insights concerning the role of carnitine in the regulation of fuel metabolism in skeletal muscle. J Physiol 581, 431-444.

47. Fischer M, Varady J, Hirche F, et al. (2009) Supplementation of L-carnitine in pigs: absorption of carnitine and effect on plasma and tissue carnitine concentrations. Arch Anim Nutr 63, 1-15.

48. Kaup D, Keller J, Most E, et al. (2018) The carnitine status does not affect the contractile and metabolic phenotype of skeletal muscle in pigs. Nutr Metab (Lond) 15, 2. 
49. Kato Y, Sugiura M, Sugiura T, et al. (2006) Organic cation/ carnitine transporter OCTN2 (Slc22a5) is responsible for carnitine transport across apical membranes of small intestinal epithelial cells in mouse. Mol Pharmacol 70, 829-837.

50. Tamai I, China K, Sai Y, et al. (2001) $\mathrm{Na}(+)$-coupled transport of L-carnitine via high-affinity carnitine transporter OCTN2 and its subcellular localization in kidney. Biochim Biophys Acta 1512, 273-284.

51. García-Delgado M, Peral MJ, Durán JM, et al. (2009) Ontogeny of $\mathrm{Na}(+) / \mathrm{L}-$ carnitine transporter and of gammatrimethylaminobutyraldehyde dehydrogenase and gammabutyrobetaine hydroxylase genes expression in rat kidney. Mech Ageing Dev 130, 227-233.

52. García-Miranda P, Durán JM, Peral MJ, et al. (2005) Developmental maturation and segmental distribution of rat small intestinal L-carnitine uptake. J Membr Biol 206, 9-16.

53. Karlic H, Lohninger A, Laschan C, et al. (2003) Downregulation of carnitine acyltransferases and organic cation transporter OCTN2 in mononuclear cells in healthy elderly and patients with myelodysplastic syndromes. J Mol Med (Berl) 81, 435-442.

54. Vaz FM \& Wanders RJ (2002) Carnitine biosynthesis in mammals. Biochem J 361, 417-429.

55. Rebouche CJ, Lehman LJ \& Olson L (1986) Epsilon-Ntrimethyllysine availability regulates the rate of carnitine biosynthesis in the growing rat. J Nutr 116, 751-759.

56. Vernez L, Dickenmann M, Steiger J, et al. (2006) Effect of L-carnitine on the kinetics of carnitine, acylcarnitines and butyrobetaine in long-term haemodialysis. Nephrol Dial Transplant 21, 450-458.

57. Primassin S \& Spiekerkoetter U (2010) ESI-MS/MS measurement of free carnitine and its precursor $\gamma$-butyrobetaine in plasma and dried blood spots from patients with organic acidurias and fatty acid oxidation disorders. Mol Genet Metab 101, 141-145.

58. Rebouche CJ (1983) Effect of dietary carnitine isomers and $\gamma$-butyrobetaine on L-carnitine biosynthesis and metabolism in the rat. J Nutr 113, 1906-1913.

59. Koeth RA, Levison BS, Culley MK, et al. (2014) $\gamma$-Butyrobetaine is a proatherogenic intermediate in gut microbial metabolism of L-carnitine to TMAO. Cell Metab 20, 799-812.

60. Rebouche CJ, Mack DL \& Edmonson PF (1984) L-Carnitine dissimilation in the gastrointestinal tract of the rat. Biochemistry 23, 6422-6426.

61. Seim H, Schulze J \& Strack E (1985) Catabolic pathways for high-dosed $\mathrm{L}(-)$ - or $\mathrm{D}(+)$-carnitine in germ-free rats? Biol Chem Hoppe Seyler 366, 1017-1021.
62. Cullinan SB, Zhang D, Hannink M, et al. (2003) Nrf2 is a direct PERK substrate and effector of PERK-dependent cell survival. Mol Cell Biol 23, 7198-7209.

63. Cnop M, Foufelle F \& Velloso LA (2012) Endoplasmic reticulum stress, obesity and diabetes. Trends Mol Med 18, 59-68.

64. Pagliassotti MJ, Kim PY, Estrada AL, et al. (2016) Endoplasmic reticulum stress in obesity and obesity-related disorders: An expanded view. Metabolism 65, 1238-1246.

65. Hoyte K, Kang C \& Martin PT (2002) Definition of pre- and postsynaptic forms of the CT carbohydrate antigen at the neuromuscular junction: ubiquitous expression of the CT antigens and the CT GalNAc transferase in mouse tissues. Brain Res Mol Brain Res 109, 146-160.

66. Thomas PJ, Xu R \& Martin PT (2016) B4GALNT2 (GALGT2) gene therapy reduces skeletal muscle pathology in the FKRP P448L mouse model of limb girdle muscular dystrophy 2 I. Am J Pathol 186, 2429-2448.

67. Kemp TJ, Sadusky TJ, Saltisi F, et al. (2000) Identification of ankrd2, a novel skeletal muscle gene coding for a stretchresponsive ankyrin-repeat protein. Genomics 66, 229-241.

68. Tsukamoto Y, Senda T, Nakano T, et al. (2002) Arpp, a new homolog of carp, is preferentially expressed in type 1 skeletal muscle fibers and is markedly induced by denervation. Lab Invest 82, 645-655.

69. Lehti M, Kivela R, Komi P, et al. (2009) Effects of fatiguing jumping exercise on mRNA expression of titin-complex proteins and calpains. J Appl Physiol 106, 1419-1424.

70. Fleischer J, Breer H \& Strotmann J (2009) Mammalian olfactory receptors. Front Cell Neurosci $3,9$.

71. Kang N \& Koo J (2012) Olfactory receptors in nonchemosensory tissues. BMB Rep 45, 612-622.

72. Pichavant C, Burkholder TJ \& Pavlath GK (2016) Decrease of myofiber branching via muscle-specific expression of the olfactory receptor mOR23 in dystrophic muscle leads to protection against mechanical stress. Skelet Muscle 6, 2.

73. Kalbe B, Osterloh M, Schulz VM, et al. (2018) OR2H2 regulates the differentiation of human myoblast cells by its ligand aldehyde 13-(13). Arch Biochem Biophys 645, 72-80.

74. Wang Z, Klipfell E, Bennett BJ, et al. (2011) Gut flora metabolism of phosphatidylcholine promotes cardiovascular disease. Nature 472, 57-63.

75. Bennett BJ, de Aguiar Vallim TQ, Wang Z, et al. (2013) Trimethylamine-N-oxide, a metabolite associated with atherosclerosis, exhibits complex genetic and dietary regulation. Cell Metab 17, 49-60. 